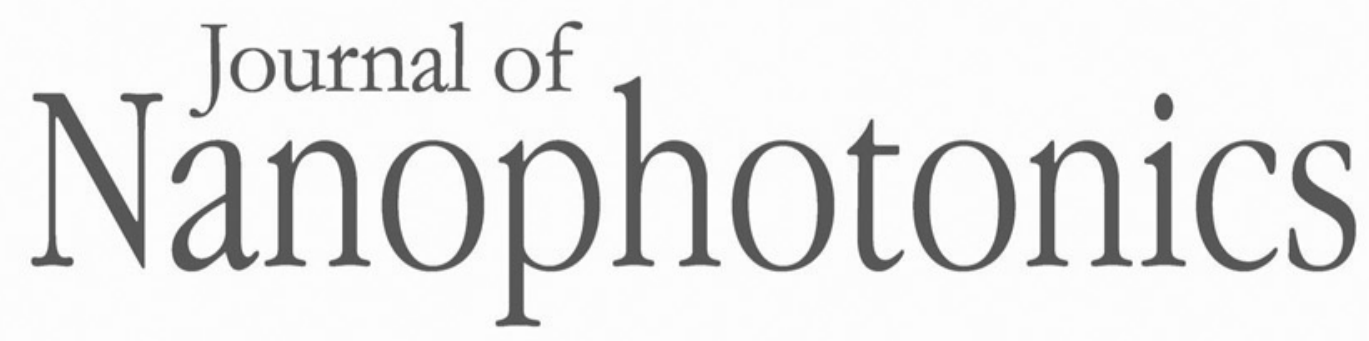

Nanophotonics.SPIEDigitalLibrary.org

\title{
Analysis of convex rib hybrid surface plasmon waveguide with ultralong propagation distance and tight mode confinement
}

Kai Zheng

Xin Zheng

Zuxun Song

\section{SPIE.}




\title{
Analysis of convex rib hybrid surface plasmon waveguide with ultralong propagation distance and tight mode confinement
}

\author{
Kai Zheng, ${ }^{\text {a,* }}$ Xin Zheng, ${ }^{\mathrm{b}}$ and Zuxun Song ${ }^{\mathrm{a}}$ \\ ${ }^{a}$ Northwestern Polytechnical University, School of Electronics and Information, No. 127, Youyi \\ West Road, Xi'an City, Shaanxi 710072, China \\ ${ }^{b}$ University of Illinois at Chicago, Department of Physics, 845 West Taylor Street, Chicago, \\ Illinois 60607, United States
}

\begin{abstract}
A hybrid surface plasmon waveguide (HSPWG) with a convex rib added on the metal surface is further investigated and analyzed theoretically. The high mode confinement and long propagation distance can be realized simultaneously by optimizing the waveguide geometry. Our designed structures can provide mode area and propagation distance achieving close to $\lambda^{2} / 512$ and $2200 \mu \mathrm{m}$ respectively, which are better than the performance of the traditional HSPWG. Our structures are excellent, promising candidates for improving plasmonic devices in the fields of ultrafast modulators and low-threshold nanolaser. (C) 2016 Society of Photo-Optical Instrumentation Engineers (SPIE) [DOI: 10.1117/1.JNP.10.016005]
\end{abstract}

Keywords: surface plasmon waveguide; mode area; propagation distance.

Paper 15157 received Nov. 4, 2015; accepted for publication Jan. 29, 2016; published online Feb. 18, 2016.

\section{Introduction}

Surface plasmon waveguides (SPWGs) have been of great interest for many years due to their excellent performance of confining the optical mode to subwavelength scale and minimizing the optical mode size by enabling the use of transverse magnetic polarization modes. ${ }^{1}$ These modes show several features such as a high proportion of mode energy localized at the interface of the dielectric layer and the metal surface and the ability of guiding mode far beyond the diffraction limit.

Although there exists excellent remarkable features, SPWGs exhibit a tradeoff between the mode confinement and propagation loss which is the result from ohmic $\operatorname{loss}^{2}$ in the metal surface. To address this issue, a so-called hybrid SPWG (HSPWG), which consists of a low-index dielectric gap embedded between the high-index dielectric and the metal layer, is theoretically proposed $^{3}$ and experimentally demonstrated. ${ }^{4}$ This new SPWG is capable of achieving large energy localization in the gap region and small propagation loss. This is because the transmission mode of HSPWG is emerged by coupling the optical mode of the high-index dielectric and the traditional surface plasmon mode created at the interface of the low-index dielectric and the metal layer.

Owing to these excellent characteristics, a variety of modified HSPWG structures such as long-range $\mathrm{HSPWG},{ }^{5}$ ridge $\mathrm{HSPWG},{ }^{6}{ }^{\mathrm{V}}$-groove $\mathrm{HSPWG},{ }^{7}$ and graphene $\mathrm{HSPWG}^{8}$ is further investigated by many research groups. These modified HSPWG structures are potentially excellent candidates exploited as directional couplers ${ }^{9}$ and polarization rotators. ${ }^{10}$ However, comparing with the traditional HSPWG, ${ }^{3,4}$ all these HSPWG structures are complicated in fabrication when achieving strong energy confinement and small propagation loss. In order to solve this issue, a modified HSPWG called convex rib HSPWG (CRHSPWG), where a convex metal rib waveguide is placed on the metal surface below the high-index dielectric cylinder waveguide, is theoretically reported. ${ }^{11}$ Its mode is two-dimensionally confined at the gap region between the

*Address all correspondence to: Kai Zheng, E-mail: zhangkai@mail.nwpu.edu.cn

$1934-2608 / 2016 / \$ 25.00$ (C) 2016 SPIE

Journal of Nanophotonics

016005-1

Jan-Mar 2016 • Vol. 10(1) 
high-index dielectric and the convex rib metal surface. However, its propagation distance is maintained at $38 \mu \mathrm{m}$, which is less than that of HSPWG with the same value of the gap height.

In this paper, the eigenmode of the CRHSPWG structures with varied gap heights and rib widths is studied using the finite element method (FEM). The trend of mode properties with varying structural parameters is shown, and the waveguide geometries leading to high mode confinement and long propagation distance are presented. The simulation results show that our structure has longer propagation distance and smaller mode confinement than that of $\mathrm{HSPWG}^{3}$ at proper range of gap region. Therefore, our designed structure has promising, excellent performance of long propagation transmission and strong energy confinement.

\section{Structures and Fabrication}

This section describes the geometry and fabrication methods of CRHSPWG. The schematic of our proposed structure is shown in Fig. 1. It consists of a submicro-Ag convex rib with width $w$ added on the $\mathrm{Ag}$ surface and a cylinder $\mathrm{Si}$ waveguide with diameter $d$ surrounded by $\mathrm{SiO}_{2}$ background.

The gap region which helps to confine the electromagnetic field in a small area is the lowindex $\mathrm{SiO}_{2}$ area embedded between the high-index $\mathrm{Si}$ cylinder and the Ag convex rib. The gap height $h$ is the distance from the lower edge of the $\mathrm{Si}$ waveguide to the upper edge of the $\mathrm{Ag}$ convex rib, whereas $h_{r}$ is the height of convex rib fixed at $50 \mathrm{~nm}$. Throughout this paper, relative permittivities of these materials are $\varepsilon_{\mathrm{Si}}=12.25, \varepsilon_{\mathrm{SiO}_{2}}=2.25$, and $\varepsilon_{\mathrm{Ag}}=-129+3.3 i^{12}$ at the telecommunication wavelength $\lambda=1550 \mathrm{~nm}$.

To fabricate such a waveguide, the metal rib can be formed by the focused-ion beam technique with relatively high precision, as demonstrated in the conventional hybrid plasmonic waveguide. ${ }^{4}$ After that, the low-index $\mathrm{SiO}_{2}$ layer is deposited on the metal rib as the gap of the waveguide, and then the high-index $\mathrm{Si}$ waveguide can be realized by the vapor-liquidsolid method. ${ }^{13}$ Although the size of the Si waveguide, the gap height, and the metal size can be controlled with high accuracy, ${ }^{4}$ our waveguide also suffers from misalignment between the Si waveguide and the metal rib. However, compared with those modified HSPWGs, ${ }^{5-10}$ our waveguide has fewer high-index nanowire layers and less complicated structures in the gap region such as wedge ${ }^{5}$ or $\mathrm{V}$-groove metal, ${ }^{7}$ which may cause less fabrication error tolerances in the real fabrication process.

\section{Results and Discussions}

For evaluating the performance of our designed CRHSPWG structures, mode properties such as the propagation distance and the normalized mode area are illustrated through varying the diameter of $\mathrm{Si}$ waveguide and the gap region. The propagation distance is defined as the distance where the initial normalized power of the guided mode decreases to $1 / e$, which can be computed as $L=\lambda / 4 \pi \operatorname{Im}\left(N_{\text {eff }}\right)$, where $\operatorname{Im}\left(N_{\text {eff }}\right)$ is the imaginary part of the complex guided mode's

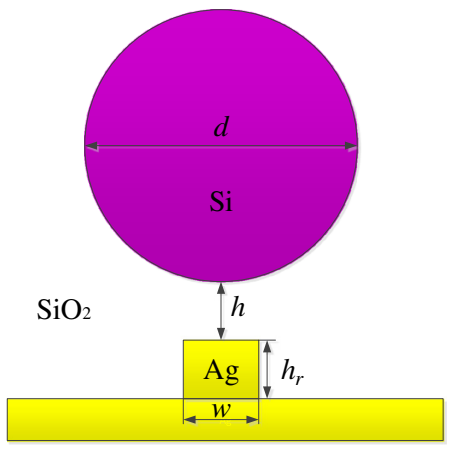

(a)

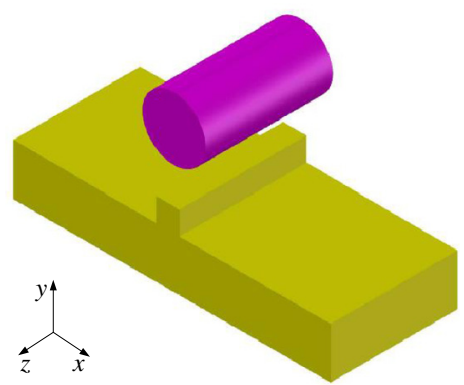

(b)

Fig. 1 Schematic of a CRHSPWG: (a) cross-sectional view, and (b) three-dimensional view. 
effective index. In order to demonstrate the mode confinement capability, the normalized mode area $M_{\text {eff }}^{3}$ is computed as

$$
\begin{gathered}
M_{\mathrm{eff}}=\frac{A_{\mathrm{eff}}}{A_{0}}, \\
A_{\mathrm{eff}}=\frac{W_{m}}{\max [W(r)]}=\frac{\int W(r) \mathrm{d}^{2} r}{\max [W(r)]},
\end{gathered}
$$

where $A_{\text {eff }}$ is defined as the ratio of total mode energy $W_{m}$ and the maximum energy density $\max [W(r)]$, and $A_{0}$ is the diffraction-limited mode area in free space expressed as $\lambda^{2} / 4$. $W(r)$ can be calculated as

$$
W(r)=\frac{1}{2}\left\{\frac{\mathrm{d}[\varepsilon(r) \omega]}{\mathrm{d} \omega}|E(r)|^{2}+\mu_{0}|H(r)|^{2}\right\}
$$

where $E(r)$ and $H(r)$ are the electrical field and magnetic field of the guided mode, respectively. In addition, to compare with the mode properties of HSPWG,${ }^{3}$ the same method-FEM-is employed to analyze CRHSPWG structures and the same results of HSPWG are obtained using our codes, which further validates our models.

The dependence of mode properties of CRHSPWG on $d$ and $h$ when setting $w$ at $50 \mathrm{~nm}$ is shown in Fig. 2. The variation of the guided mode's property in CRHSPWG follows that of HSPWG, while gradually increasing $d$ from 100 to $500 \mathrm{~nm}$ with different $h$. As illustrated in Fig. 2, owing to the small gap region of CRHSPWG, we can find some ranges of $d$ and $h$ where our designed waveguide can provide an order of smaller mode area or longer propagation distance than that of HSPWG. More specifically, the smallest mode area and the longest propagation distance of CRHSPWG approach to $\lambda^{2} / 512$ and $2000 \mu \mathrm{m}$, while keeping $d=200 \mathrm{~nm}, h=10 \mathrm{~nm}$ and $d=500 \mathrm{~nm}, h=10 \mathrm{~nm}$, respectively. For small diameter $d$ and gap height $h$ (e.g., $d=150 \mathrm{~nm}, h=10 \mathrm{~nm}$ ), high electromagnetic energy is localized at the interface of $\mathrm{Ag}$ and $\mathrm{SiO}_{2}$, as shown in Fig. 2(c), which is resulted from the surface plasmon

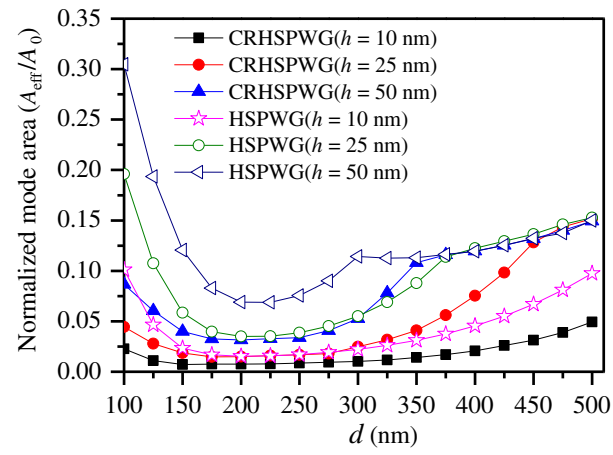

(a)

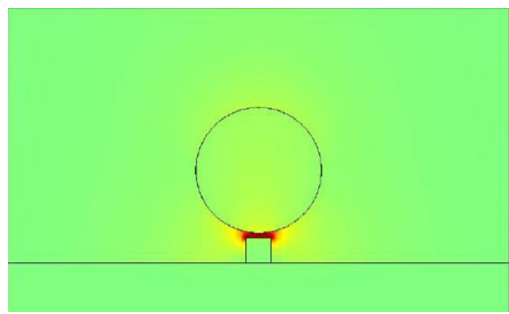

(c)

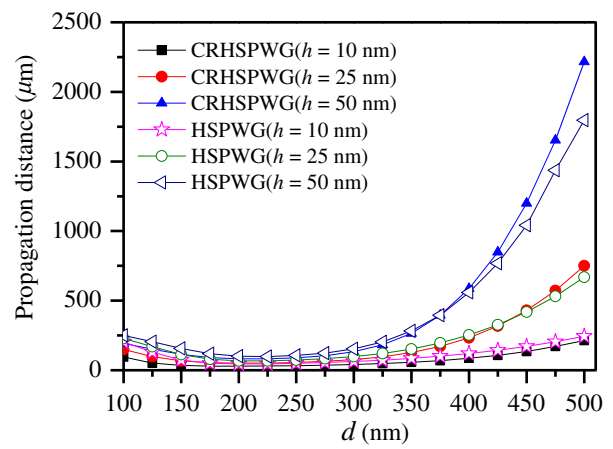

(b)

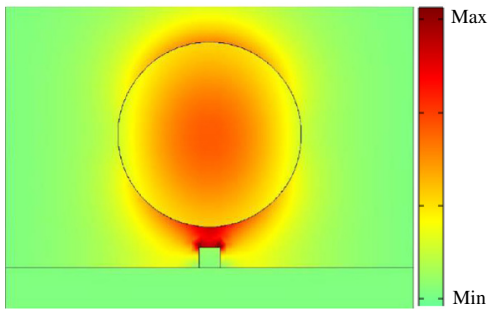

(d)

Fig. 2 Mode properties versus $d$ and $h$ while setting $w$ at $50 \mathrm{~nm}$ : (a) normalized mode area, and (b) propagation distance. (c) and (d) Normalized electromagnetic energy distributions for typical structures: (c) $[d, h]=[150,10] \mathrm{nm}$, and (d) $[d, h]=[450,50] \mathrm{nm}$. 
polarization (SPP)-like mode emerged in CRHSPWG. On the other hand, for large diameter $d$ and gap height $h$ (e.g., $d=450 \mathrm{~nm}, h=50 \mathrm{~nm}$ ), the cylinder-like mode is supported by CRHSPWG where a large part of energy exists in Si waveguide as illustrated in Fig. 2(d).

To gain a deeper understanding of the hybrid mode in CRHSPWG, the mode character is calculated using the coupled-mode theory, ${ }^{3}$ as shown in Fig. 3. An approximation hybrid mode and its mode character are calculated by a set of equations:

$$
\begin{gathered}
\varphi_{ \pm}(d, h)=a_{ \pm}(d, h) \varphi_{\mathrm{cyl}}(d)+b_{ \pm}(d, h) \varphi_{\mathrm{spp}}, \\
b_{ \pm}(d, h)=\sqrt{1-\left|a_{ \pm}(d, h)\right|^{2}}, \\
\left|a_{ \pm}(d, h)\right|^{2}=\frac{n_{\mathrm{hyb}}(d, h)-n_{\mathrm{spp}}}{\left[n_{\mathrm{hyb}}(d, h)-n_{\mathrm{cyl}}(d)\right]+\left[n_{\mathrm{hyb}}(d, h)-n_{\mathrm{spp}}\right]},
\end{gathered}
$$

where $\varphi_{ \pm}(d, h)$ is the strength of the hybrid mode, $a_{ \pm}(d, h)$ and $b_{ \pm}(d, h)$ are the amplitudes of the cylinder mode $\varphi_{\text {cyl }}(d)$ in Si waveguide and of the SPP mode $\varphi_{\text {spp }}$ in $\mathrm{Ag}-\mathrm{SiO}_{2}$ interface, respectively. In Eq. (6), $n_{\mathrm{hyb}}(d, h)$ and $n_{\mathrm{spp}}$ are the mode effective indices of $\varphi_{\mathrm{cyl}}(d)$ and $\varphi_{\mathrm{spp}}$, respectively. It is obvious that when $n_{\mathrm{hyb}}(d, h)>n_{\mathrm{spp}}$, which leads to the mode character $\left|a_{ \pm}(d, h)\right|^{2}>0.5$, a large portion of energy is located in the cylinder $\mathrm{Si}$ waveguide, which makes our hybrid mode a cylinder-like mode. On the contrary, a large amount of energy exists in $\mathrm{Ag}-\mathrm{SiO}_{2}$ interface, which lets our hybrid mode be SPP-like mode. From Fig. 3, one can easily tell that the critical diameter $d$ is obtained at $200 \mathrm{~nm}$ when setting $\left|a_{ \pm}(d, h)\right|^{2}=0.5$ and increasing $h$ can accelerate hybrid mode to be more cylinder-like mode. These can be used to theoretically explain why our CRSPWG mode properties are changed when varying $d$ and $h$ as plotted in Fig. 2 .

Figure 4 displays the dispersion relation of CRHSPWG with different $d$ and $h$. Due to the bounded nature of SPP mode, the guided mode of CRHSPWG excitations corresponds to the dispersion curve lying to the right of the respective light line of $\mathrm{SiO}_{2}$. On the other hand, the guided mode's dispersion curve lying to the left of light line of $\mathrm{Si}$ has more property of cylinder mode when the frequency or diameter $d$ becomes larger. The gap height $h$ also plays an enhanced role in the determination of dispersion curves while $h$ is small (e.g., $h=200 \mathrm{~nm}$ ).

In order to further investigate the gap region's influence in the performance of CRHSPWG, the dependence of mode properties of CRHSPWG with varied $w$ and $h$ is compared with the HSPWG, as shown in Fig. 5. The diameter of Si waveguide $d$ is set at $450 \mathrm{~nm}$, where the tight mode confinement and long propagation distance are obtained from Fig. 2. For a small $h$ (e.g., $h<25 \mathrm{~nm}$ ), there exists a range of $w$ where the normalized mode area of CRHSPWG is smaller than its HSPWG counterpart due to the smaller gap region of CRHSPWG. As further increasing $h$ to $50 \mathrm{~nm}$, the enlarged gap region starts to hold pure cylinder-like modes, which leads more areas to be nearly stable. Moreover, it is interesting to note that for a small $w$ (e.g., $w<30 \mathrm{~nm}$ ), the radiation loss occupies the main part of the propagation loss, which is smaller than that of

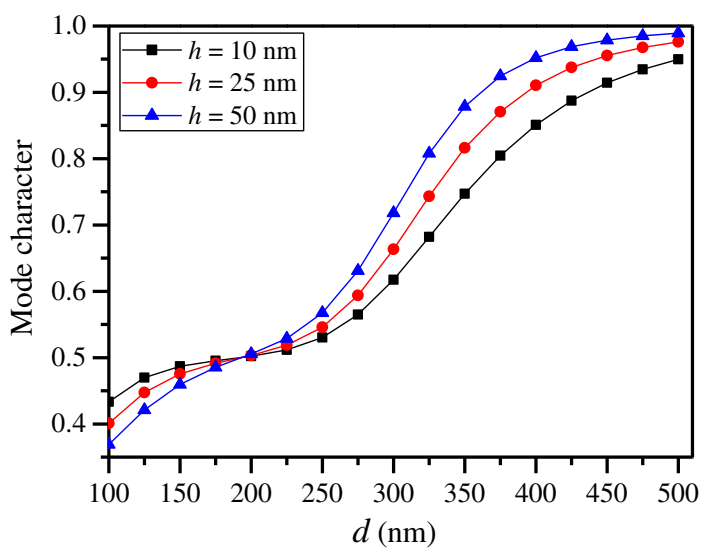

Fig. 3 Mode character versus $d$ and $h$ while setting $w$ at $50 \mathrm{~nm}$. 
Zheng, Zheng, and Song: Analysis of convex rib hybrid surface plasmon waveguide...

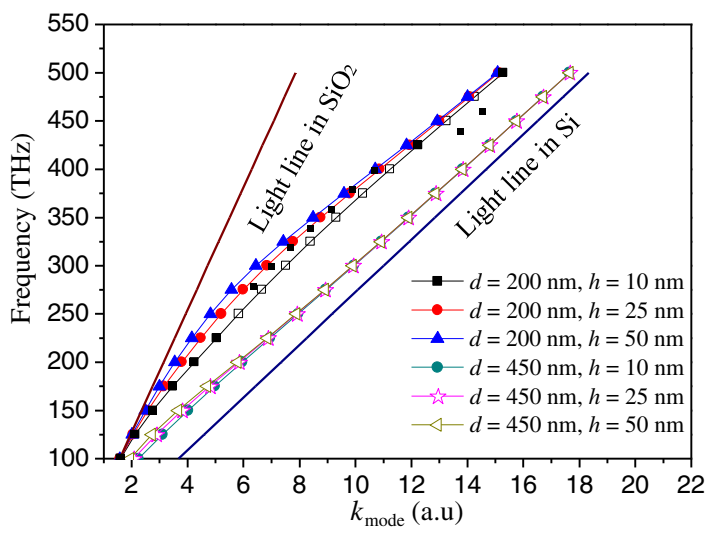

Fig. 4 Dispersion relation with different $d$ and $h$, where $k_{\text {mode }}$ is the wave number of guided mode.

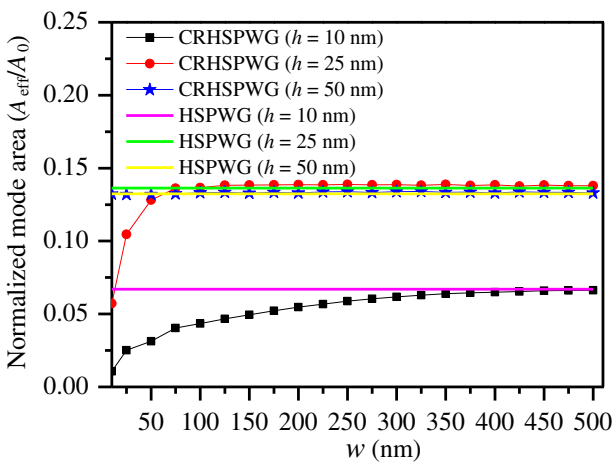

(a)

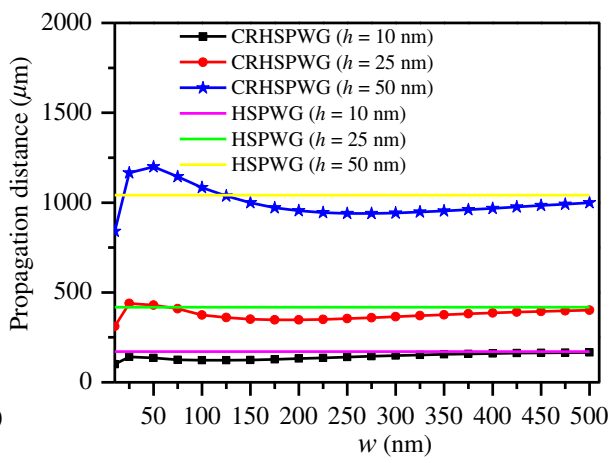

(b)

Fig. 5 Mode properties versus $w$ and $h$ while setting $d$ at $450 \mathrm{~nm}$ : (a) normalized mode area, and (b) propagation distance.

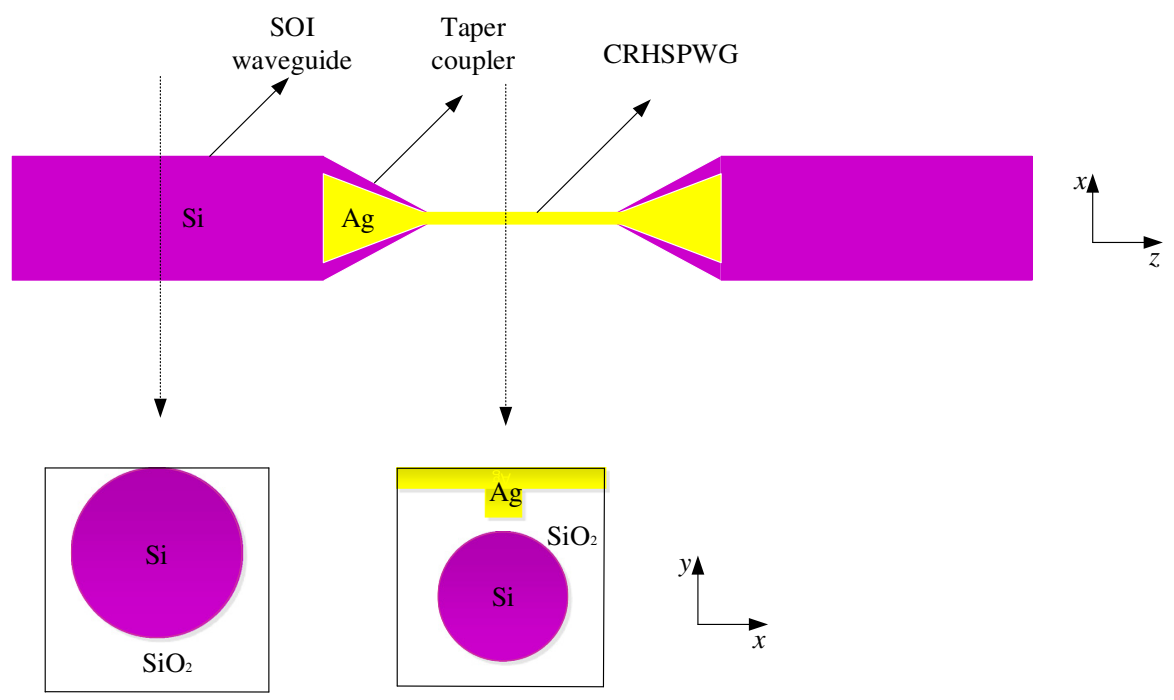

Fig. 6 Schematic of a taper coupler structure based on CRHSPWG, where the cladding Ag layer is not depicted for xoz plane. 
HSPWG with the same parameters. However, for a large $h$ (e.g., $h>25 \mathrm{~nm}$ ), we can find a range of $w$ where the propagation distance of CRHSPWG is longer than its HSPWG counterpart, owing to the smaller interface of $\mathrm{Ag}$ in $\mathrm{SiO}_{2}$ layer. As $w$ is further increased, the metal loss surmounts the radiation loss in CRHSPWG and leads to a shorter propagation distance than its HSPWG counterpart.

To achieve the goal of on-chip telecommunication applications, our CRHSPWG is used to connect two identical dielectric waveguides, which leads to the coupling between the surface plasmon mode and the photonic mode in communication. Therefore, an experimentally demonstrated coupler structure ${ }^{14}$ is designed to support highly efficient coupling between CRHSPWG and Si photonic waveguides on the same silicon-on-insulator (SOI) chip, as shown in Fig. 6. This structure consists of SOI waveguide, CRHSPWG, and metallic tapered coupler, which is capable of converting photonic mode in SOI waveguide to surface plasmonic mode. In future research, we will further calculate the parameters of couplers and prepare for fabrication.

\section{Conclusion}

In this paper, we further investigate the CRHSPWG based on FEM method. To better understand the performance of our structures, the simulation results are compared with those of the traditional hybrid plasmonic waveguides. Our simulation results show that through varying the gap region, waveguide structures have the capability of supporting longer propagation distance and smaller mode area than those of $\mathrm{HSPWG}^{3}$ with the same value of $h$. More specifically, $h$ and $d$ of CRHSPWG play critical roles in determining whether the characteristics of guided coupled mode behave as the SPP-like mode or cylinder mode. While setting $h$ and $d$ in a suitable range, varying $w$ efficiently reduces the mode area or increases the propagation distance. In addition, a tapered coupling structure based on CRHSPWG is further designed to realize on-chip telecommunication in lab fabrication. Therefore, changing the gap region of HSPWG remains the most critical issue for obtaining long propagation transmission and high energy confinement in the fields of ultrafast modulators and low-threshold nanolaser.

\section{Acknowledgments}

The authors would like to acknowledge Professor Olivier J. F. Martin at Duke University and Doctor Qi Dai at University of Illinois at Urbana-Champaign for useful references and simulation discussions.

\section{References}

1. S. A. Maiera and H. A. Atwater, "Plasmonics: localization and guiding of electro-magnetic energy in metal/dielectric structures," J. Appl. Phys. 98, 011101 (2005).

2. A. V. Zayats, I. I. Smolyaninov, and A. A. Maradudin, "Nano-optics of surface plasmon polaritons," Phys. Rep. 408(3-4), 131-314 (2005).

3. R. F. Oulton et al., "A hybrid plasmonic waveguide for subwavelength confinement and long-range propagation," Nat. Photonics 2, 496-500 (2008).

4. R. F. Oulton et al., "Plasmon lasers at deep subwavelength scale," Nature 461, 629-632 (2009).

5. Z. Zhang and J. Wang, "Long-range hybrid wedge plasmonic waveguide," Sci. Rep. 4, 6870 (2014).

6. C. C. Huang, "Metal nanoridges in hollow Si-loaded plasmonic waveguides for optimal mode properties and ultra-compact photonic devices," IEEE J. Sel. Top. Quantum Electron. 20(4), 600409 (2014).

7. C. Zhaoxian et al., "Hybrid plasmonic waveguide in a metal V-groove," AIP Adv. 4, 017103 (2014).

8. Q. M. Zhang et al., "Graphene surface plasmons at the near-infrared optical regime," Sci. Rep. 4, 6559 (2014). 
9. J. H. Choe and J. T. Kim, "Analysis of polarization-splitting characteristics in three-corebased directional couplers using silicon hybrid plasmonic waveguides," J. Lightwave Technol. 33(10), 2099-2105 (2015).

10. Y. Xu, J. Xiao, and X. Sun, "A compact hybrid plasmonic polarization rotator for siliconbased slot waveguides," IEEE Photonics Technol. Lett. 26(16), 1609-1612 (2014).

11. R. Hao, E. Li, and X. Wei, "Two-dimensional light confinement in cross-index modulation plasmonic waveguides," Opt. Lett. 37(14), 2934-2936 (2012).

12. P. B. Johnson and R. W. Christie, "Optical constants of the noble metals," Phys. Rev. B 6, 4370-4379 (1972).

13. T. Kuykendall et al., "Crystallographic alignment of high-density gallium nitride nanowire arrays," Nat. Mater. 3, 524-528 (2004).

14. W. Marcelo, H. Zhanghua, and V. Vien, "Conductor-gap-silicon plasmonic waveguides and passive components at subwavelength scale," Opt. Express 18(11), 11728-11735 (2010).

Kai Zheng received his BS degree in electronic and information engineering and his MS degree in electromagnetic field and microwave technology from Northwestern Polytechnical University, Xi' an City, China, in 2008 and 2012, respectively. He is currently a PhD student at the School of Electronics and Information at Northwestern Polytechnical University. His current research interests include plasmonics, nanolasers, and optical sensors.

Xin Zheng received her BS degree in material science and engineering and her MS degree in material science from Northwestern Polytechnical University, Xi' an City, China, in 2008 and 2012, respectively. She is currently a PhD candidate at the Department of Physics, University of Illinois at Chicago, Chicago, Illinois, USA. Her current research interests include the MBE growth, characterization, and device fabrication of $\mathrm{HgCdTe}$ and $\mathrm{CdMnTe}$.

Zuxun Song is currently a professor with the 365 Institute, Northwestern Polytechnical University, Xi' an City, China. He received his PhD in 2004 from the School of Electronics and Information, Northwestern Polytechnical University. His current research interests include microwave instrumentation, microwave communication, and electromagnetic compatibility. 